\title{
Forsen Herbal Tea in Reserpine in Mice Model of Spleen Deficiency of Gastrointestinal Effects
}

\author{
Wang Ting ${ }^{1}$, Miao mingsan ${ }^{1, *}$, Li Yan ${ }^{1}$, Wang Tan ${ }^{1}$, Bai Ming ${ }^{1}$, Wang Zuhong ${ }^{2}$ \\ ${ }^{1}$ Henan University of Traditional Chinese Medicine, China Zhengzhou, 450000 \\ ${ }^{2}$ Fusen Group, China Nanyang, 473000 \\ aemail: wangtingyaoli@foxmail.com, "corresponding author bemail: Miaomingsan@163.com,
}

Keywords: Forsen source herbal tea; bioelectricity and organ morphology; reserpine

\begin{abstract}
To observe the Forsen source herbal tea of reserpine in mice model of spleen deficiency of gastrointestinal effects. Mice were injected with reserpine injection to each mice by $0.1 \mathrm{mg} / \mathrm{kg}$ in 17 days with continuous subcutaneous injection molding, mice dranking water solution respectively at the same time, pills, small dose, small dose of Fukumori Genryo tea, Wang Laoji, will be the corresponding herbal tea instead of mice daily drinking water; the control group was given ordinary drinking water. In 16th, mice were measured the gastrointestinal bioelectric and sacrificed at 17th. To observe the mice's bioelectricity and organ morphology changes. the spleen deficiency of mice model, compared with the model group, the large and small dose of the Forsen source herbal tea in mice bioelectricity and tissuse morphology without large the influence of the Forsen source herbal tea. No obvious adverse effect on spleen deficiency mice induced by reserpine bioelectricity and organ morphology.
\end{abstract}

\section{Introduction}

From honeysuckle, licorice, immortality, dried ginger, chrysanthemum, radix puerariae, hawthorn made of Forsen source herbal tea. The herbs are containing components cool. For herbal tea containing more than cool traditional Chinese medicine, whatever the weakness of the spleen and stomach can drinke Forsen source herbal tea.With the increase of modern work pressure, people have irregular diet to gastrointestinal function disorder. Drinking a lot of Forsen source herbal tea whether will lead to the aggravation of the gastrointestinal weak [1], so therefore observed long-term drinking a lot of Forsen source herbal tea in in mice model of spleen deficiency of gastrointestinal. Therefore, we had to observe long-term drinking lots of Forsen source herbal of injection of reserpine induced mice with spleen deficiency model.

\section{Experimental Material}

Pharmaceutical companies to provide cool tea: from honeysuckle, chrysanthemum, grass jelly, licorice, dried ginger, radix puerariae, hawthorn, Chinese yam and other Forsen source herbal tea; Wong Lo Kat herbal tea, batch number 20140901 (specifications 310ml/ bottle); reserpine injection (1ml:1mg), Tianjin Jinyao Pharmaceutical Co., Ltd., batch number 1501091.

Gastrointestinal biological apparatus: EGEG-2D type detection apparatus, Hefei Austrian Science and Technology Development Co., Ltd.

Kunming mice, male and female, weighing $18 \sim 22 \mathrm{~g}$; experimental animal center of Shandong province quality certificate: No 37005400000013 ;

\section{Experimental methods}

Take the weight of 18 to $8422 \mathrm{~g}$ mice, male and female, were randomly divided into 7 groups, 6 groups of these mice were spleen and deficiency model, another group was control group. Model group were injected with reserpine injection per mouse by $0.1 \mathrm{mg} / \mathrm{kg}$ in subcutaneous injection, until continuous 17 days. Mice were given 6 groups respectively forsen source a large dose of herbal tea, a small dose of herbal tea (small dose concentration is half of large dose concentration), a large dose of Wang Laoji, a small dose of Wang Laoji (small dose concentration is half of large 
dose concentration), Baohewan aqueous solution that will replace the corresponding herbal tea drinking water of mice; blank group were given normal drinking water. The mice drank corresponding herbal tea instead of drinking water. Mice changed daily drinking water bottle contents every day, continuous 17 th. In 16th, mice were anesthetized with ether, in the gastric antrum surface projection of the shearing, 95\% B Alcohol degreasing with disking electrode contained saline tampon cover and fixed; The left forelimb wrist as reference electrode and left hind tarsal on as the ground electrode. EGEG-2D gastrointestinal electrographic instrument were recorded $5 \mathrm{~min}$ electrogastrogram. Observe the effect on gastrointestinal motility and outcome measures mainly frequency and amplitude. In 17th mice were killed. To put the mice's stomach (cut along the greater curvature of the stomach and contents make full use of saline wash) and duodenum. Then the stomach and duodenum tissue was placed in $10 \%$ neutral buffered formaldehyde solution fixed $24 \mathrm{~h}$, making up by slicedand and microscopic examination.

\section{Results}

Table 1 Effect of Forsen source herbal tea gastrointestinal bioelectric in mice $(\bar{x} \pm s)$

\begin{tabular}{cccc} 
Group & $\mathrm{n}$ & Frequency (times/min) & Amplitude (mv) \\
Blank group & 12 & $3.11 \pm 0.17^{* *}$ & $114.06 \pm 10.41^{* *}$ \\
Model group & 12 & $2.74 \pm 0.20$ & $92.64 \pm 11.22$ \\
Baohewan group & 12 & $3.02 \pm 0.16^{* *}$ & $108.15 \pm 10.95^{* *}$ \\
Large dose group forsen herbal tea & 12 & $2.95 \pm 0.19^{* *}$ & $102.72 \pm 10.40^{*}$ \\
small dose group forsen herbal tea & 12 & $2.95 \pm 0.25^{* *}$ & $102.02 \pm 11.03^{*}$ \\
Large dose group Wang Laoji & 12 & $2.83 \pm 0.18$ & $96.76 \pm 10.60$ \\
small dose group Wang Laoji & 12 & $2.90 \pm 0.21$ & $98.72 \pm 10.53$ \\
\hline
\end{tabular}

* Indicates that the model group is more than $* * \mathrm{P}<0.01, \mathrm{P}<0.05$

From the table we can see that with the blank group, model group were significantly decreased ( $\mathrm{P}<$ 0.01) with frequency and number of gastrointestinal biological electricity. Compared with the model group, small dose group and herbal tea Fusen; Baohewan group are mouse gastrointestinal bioelectric frequency increased slightly $(\mathrm{P}<0.01)$; large and small doses of Wang Laoji group mice on gastrointestinal bioelectric frequency was not significantly increased. Baohewan group increased significantly in mice gastrointestinal bioelectric amplitude $(\mathrm{P}<0.01)$. Large and small doses of Forsen source herbal group increased significantly in mice gastrointestinal bioelectric amplitude $(\mathrm{P}<0.05)$. Large and small dose group although Wang Laoji herbal tea mice gastrointestinal bioelectric the amplitude increased slightly, but there are obvious differences were not found. A large number of long-term drinking herbal tea tips on normal gastrointestinal function of mice's basic no obvious adverse effects.

\section{Histopathological observation of gastric mucosa:}

From the table 2 can be seen, the blank control group mice gastric mucosa had no obvious abnormalities; a large number of long-term drinking herbal tea will has a certain effect on the mice gastric mucosa, gastric mucosa occasionally part of the region appeared in principal cells, reduce the number of cell wall, reduce the number of gastric body;, small dose Forsen source herbal tea mice gastric mucosa of the relative small change; change of big and small dose of Wong Lo Kat mice gastric mucosa is relatively large. The Ridit test, with the blank control group, each group of data without significant difference that Forsen source herbal tea on the gastrointestinal function of mice with spleen deficiency without obvious adverse effects. 
Table 2 Effect of Forsen source herbal reserpine in mice gastric mucosa

\begin{tabular}{|c|c|c|c|c|c|}
\hline Group & $\mathrm{n}$ & - & + & ++ & +++ \\
\hline Blank group & 12 & 12 & 0 & 0 & 0 \\
\hline Model group & 12 & 0 & 1 & 2 & 8 \\
\hline Baohewan group & 12 & 9 & 2 & 1 & 0 \\
\hline Large dose group forsen herbal tea & 12 & 4 & 1 & 2 & 5 \\
\hline small dose group forsen herbal tea & 12 & 2 & 3 & 2 & 5 \\
\hline Large dose group Wang Laoji & 12 & 1 & 3 & 2 & 6 \\
\hline small dose group Wang Laoji & 12 & 2 & 3 & 1 & 6 \\
\hline
\end{tabular}

"-"The principal cells of the gastric mucosa, cell wall, gastric body normal number; "+" gastric mucosa gland epithelial cells with mild edema, reduce the number of gastric glands. "++" gastric mucosa occurred in the local region of principal cells, reduce the number of cell wall, gastric mucosa epithelial cells edema, occasionally gastric a reduction in the number. " +++ " of gastric glands in principal cells, reduce the number of cell wall, gastric mucosa epithelial cells edema obviously, glandular atrophy and with the number of reduced.

\section{Observation of gastric mucosa:}

The control group of mice gastric mucosa structural integrity of epithelial cells arranged orderly, glandular epithelium normal, lamina propria no congestion and inflammatory cell infiltration, muscularis mucosa integrity, see Fig 1; stomach spleen deficiency model group mice than normal mice gastric mucosa cells of the gland main thin, visible, cell wall in order to reduce the number of cells reduced, part of gastric mucosal epithelial cells edema, glandular atrophy and with the decrease in the number of cells, see Fig 2 pills and mice gastric mucosa thickness and gastric mucosal epithelial cells in the cell wall, and returned to the level of normal mice, no gland atrophy, see Fig 3 herbal tea the large dose group of mice gastric mucosa occasionally part of the main cell wall, cell number reduced, reducing the number of gastric glands, see Fig 4 herbal tea small dose group and model group compared with spleen and stomach deficiency, a part of the region Now principal cells, reduce the number of cell wall, reducing the number of gastric and see Figs 5. Wong Lo Kat large dosage group of gastric mucosal epithelium cells in the diffuse edema, part of the region appeared in principal cells, reduce the number of cell wall and with glandular atrophy, see Figs. Wong Lo Kat light of large dose of lesions of the small-dose group in gastric mucosa epithelial cells edema group, chief cells, parietal cell number decreased obviously, occasionally part of glandular atrophy, see Fig7.

\section{Observation of mouse gastric tissue by light microscope:}

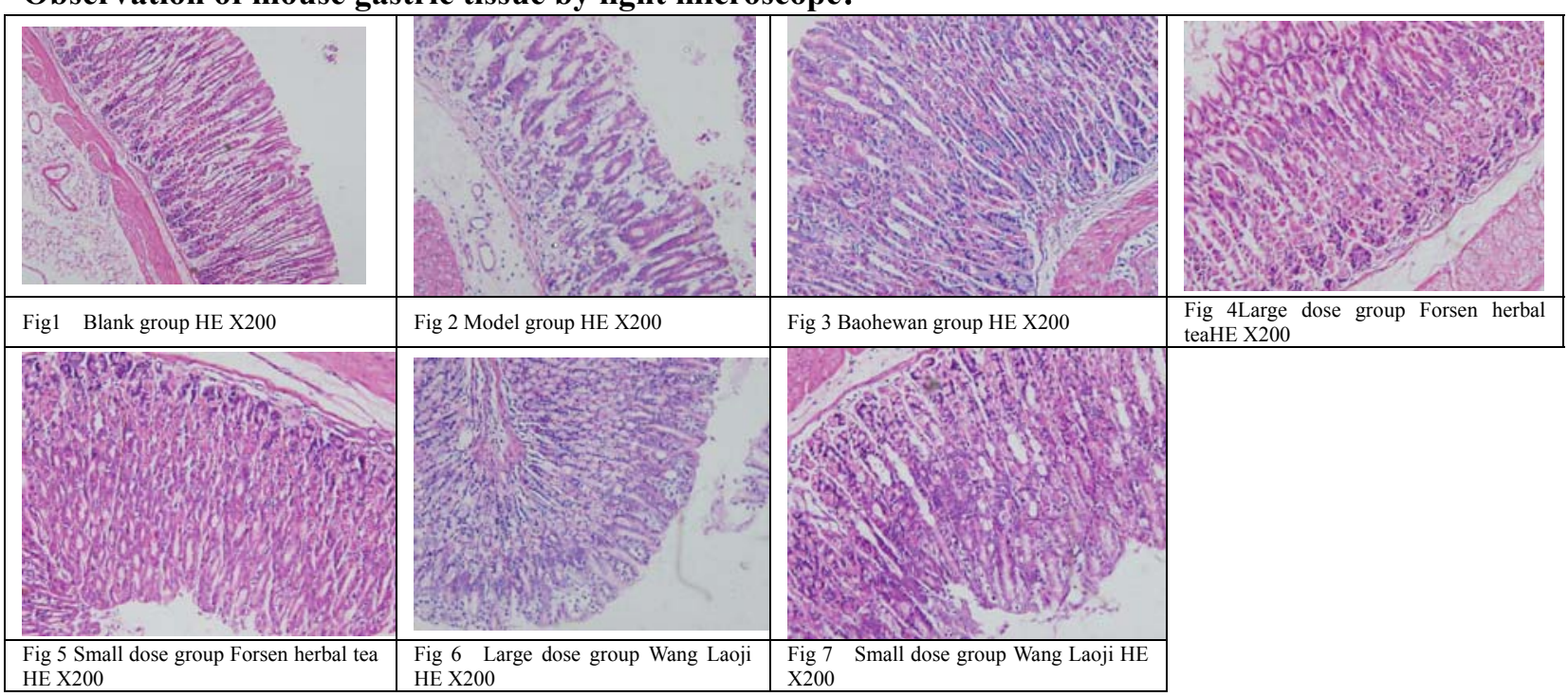


Histopathological observation of duodenum:

Table 3 Observation on reserpine in mice duodenum Forsen source herbal histopathology

\begin{tabular}{cccccc} 
Group & $\mathrm{n}$ & - & + & ++ & +++ \\
\hline Blank group & 12 & 12 & 0 & 0 & 0 \\
Model group & 12 & 0 & 2 & 1 & 9 \\
Baohewan group & 12 & 8 & 4 & 0 & 0 \\
Large dose group Forsen herbal tea & 12 & 3 & 2 & 3 & 4 \\
small dose group Forsen herbal tea & 12 & 2 & 3 & 3 & 4 \\
Large dose group Wang Laoji & 12 & 1 & 2 & 3 & 6 \\
small dose group Wang Laoji & 12 & 1 & 3 & 2 & 6
\end{tabular}

"-": Normal small intestinal mucosa epithelial; "+": The small intestine tissue appeared in the apical epithelial cell degeneration and necrosis of the small intestine; "+ +": Intestinal tissue local intestinal villus epithelial cell degeneration and occasional necrosis. "++ +": Small intestinal mucosa significantly thinner, and shortening of villus and accompanied by loss, most tip of villus epithelial cell degeneration or focal necrosis.

From the table 3 can be seen, the duodenal tissue of mice in the normal group were normal, a large number of long-term drinking herbal tea will of duodenal tissue in mice with spleen deficiency local intestinal villus epithelial cell degeneration and occasional necrosis, large, small dose Forsen source herbal tea group mice symptoms lighter, large and small dose of Wong Lo Kat group of mouse duodenal symptoms heavier. The Ridit test, with the blank control group, each group of data without significant difference that Forsen source herbal tea to the normal gastrointestinal function of mice without obvious adverse effects.

Observation of gastric mucosa:

Normal control mice intestinal mucosa of normal villus shedding phenomenon, without any distortion or focal necrosis, see Fig 8; in the model group than the control group of mice intestinal mucosa was thinned, villus shortening and accompanied by shedding most of the top, villous epithelial cell degeneration or necrosis, see Figs 9; small intestinal mucosa of mice in Baohewan group restored to normal level, see Fig 10; large dose group local herbal tea Forsen source tip of villus epithelial cell degeneration and occasional necrosis, see Fig 11; slight tip of villus epithelial cell degeneration appeared in small dose Fu Mori Ryocha group, no focal necrosis, see Fig 12 big; dose Wang Laoji mice villus epithelial cell degeneration and focal necrosis of model group is serious, part of the intestinal villi off, see Fig 13; small group of small dose of Wang Laoji The villus epithelial cells of focal necrosis than large dose group Wanglaoji Fig 14.

Observation of mouse duodenal tissue under light microscope:

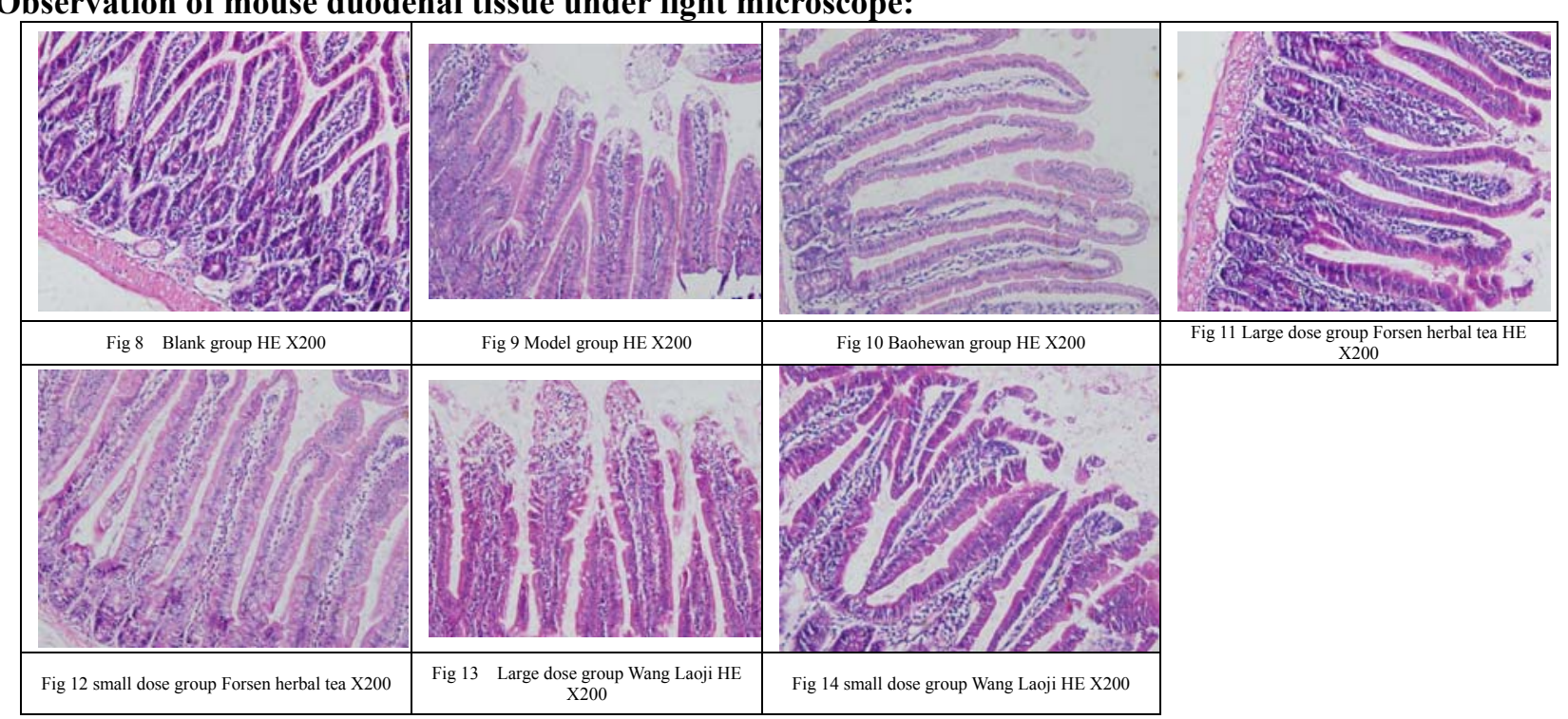




\section{Conclusion}

Gastrointestinal motor function is an important part of the physiological function of the digestive tract. The dysfunction is the cause of gastrointestinal symptoms in one of the decisive factors [5.8]. For gastrointestinal motor function most can express is gastrointestinal bioelectric expression. Normal people use a lot of cool traditional Chinese medicine may lead to gastrointestinal function disorder, gastrointestinal disorders will lead to people eating and elimination of abnormal, [7.10] is often accompanied by insomnia, anxiety, attention and lax wait for a phenomenon, hence the observed long-term drinking a lot of Forsen herbal tea on the body weight of normal mice, drinking water and feed amount and biological changes of whether there is the influence.

Experiments show that, compared with the model group, small dose group herbal tea Fusen large dose group Forsen herbal tea and Baohewan group of mice were gastrointestinal bioelectric frequency increased slightly $(\mathrm{P}<0.01)$; large and small doses of Forsen herbal tea group increased significantly in mice gastrointestinal bioelectric amplitude $(\mathrm{P}<0.05)$, basically no obvious adverse effects for a long time drinking tea on the gastrointestinal function of normal mice. Histological observation can be seen, a large number of long-term drinking herbal tea on gastric mucosa of mice were occasionally part of the main cell wall, cell number reduced, reducing the number of gastric glands; but, small dose group of mice gastric mucosa Forsen herbal tea change is relatively small; after the examination, and the blank compared with the control group, there is no significant difference between groups of data, the normal mice duodenum tissues were normal, a large number of long-term drinking herbal tea on duodenal tissue in mice with spleen deficiency syndrome occurred in the tip of villus epithelial cell degeneration occasionally Necrotic, big, small dose of herbal tea group were mild; large and small dose group of mice duodenum Wong Lo Kat severe symptoms. By Ridit test, compared with the blank control group, there is no significant difference between groups of data, basic no obvious adverse effects on gastrointestinal function in normal mice Forsen herbal tea. Observation of mouse gastric tissue by light microscope: Large and small dose group of local Forsen herbal tea tip of villus epithelial cell degeneration and occasional necrosis; control group of mice gastric mucosa structural integrity of epithelial cells arranged orderly, glandular epithelium normal, lamina propria no congestion and inflammatory cell infiltration, gastric muscularis mucosa integrity; spleen deficiency model group mice than in normal gastric mucosa mice thin, main cells of the gland cell wall, to reduce the number of cells reduced, part of gastric mucosal epithelial cells edema, glandular atrophy and with reduced number Less; the experimental results show that the long-term consumption ofForsen herbal teafor mice has no adverse effects on gastrointestinal effects.

Although Forsen herbal tea had cold traditional Chinese medicine [9], but according to the pathological changes of spleen deficiency mice gastrointestinal bioelectric and gastrointestinal tissues. It is proved that theForsen herbal teafor spleen deficiency mice did not produce significant adverse impact. At the same time, the experiment for Forsen herbal tea production in provides the theoretical and experimental basis.

\section{References}

[1] Miao Ming three, Zhou Hongyan, Li Ping ruffian. Full Xiao granule effect on gastrointestinal peristalsis of mice [J],.2000.2,13 traditional Chinese Medicine Research (1): 34-35.

[2] Wang Lan, Zhang Xiaowen, Zhao Guangcai, Wang Yu. Review of the efficacy and safety of herbal tea drinks herbal tea (5): 914-916.

[3]Zhang Shuping, Guangzhou [J]. Henan.2005:25 Chinese herbal tea on the pros and cons (9): 78

[4] Li Chen, Wang Chuijie. Comparison of gastric mucosal damage in rats with spleen deficiency syndrome (3) [J].:127-129. (3).2013,21

[5] Fang Xiong Wu, Hongyan, Hainan and rhubarb on plasma of rats with spleen qi deficiency growth chalone and gastric mucosal injury [J]. Chinese medicine forum [J].2010,25 (1): 35-37. 
[6] Manuela in Pan Zhiqiang, coincident Hou Li. Different methods induced experimental gastric ulcer in mice of different syndromes in the same disease [J]. Journal of Hunan Chinese medicine. 2008,24 (1): 70-78.

[7] Xu Liuqing, Qin Lin, Jia Feng Wang, Qu Xiaoni. Bitter cold medicine on gastrointestinal function influence research survey [J]. Shandong University of traditional Chinese Medicine Journal of.2011:34 (6), 554-556.

[8] kege Mae Philippines, Wang Lanqing. Traditional Chinese medicine Xiao Ji powder external application on gastrointestinal dynamics [J]. Chinese medicine pediatrics. 2009: 1 (02): 130-132.

[9] Guo Yifeng, Zhou Wenli, Zhang Jianpeng, Liu Junhua, Feng Wei Hua, Jiao BH. Cuttlebone polysaccharide on mouse gastric mucosal protective effect [J]. The Second Military Medical University Journal of.2008:25 (11). 1328-1332

[10] Hershey P, Xi Ping, Liao Xue Zhen, Chen muyuan. Experimental study on the f.hirta Decoction of rhubarb spleen deficiency gastrointestinal motor function in mice [J]. Pharmacy today, 2012:22 (7):398-401. 